\title{
Keine Haftung des Arztes trotz groben Behandlungsfehlers Patient widersetzt sich dringenden ärztlichen Empfehlungen
}

Entgegen der üblichen Regel, dass sich die Beweislast bei einem groben Behandlungsfehler zu Lasten des Arztes umdreht, gilt dies nicht, wenn massives Eigenverschulden des Patienten den Heilungserfolg gefährdet.

\section{Der Fall}

Der Kläger war Berufsfußballer. Während eines Spiels erlitt er am 31.10.2004 eine Bissverletzung, die im weiteren Verlauf zu einer Kniegelenksinfektion führte. Bei einem heftigen Zweikampf hatten die Schneidezähne seines Gegenspielers eine Rissverletzung am rechten Knie des Klägers verursacht.

Nach dem Zusammenprall eilte der (später verklagte) Mannschaftsarzt auf den Platz und übernahm die Erstversorgung in der Mannschaftskabine. Er desinfizierte die Risswunde im Bereich des Knies bei sparsamer Wundrandkorrektur und nähte die Verletzung. Der Mannschaftsarzt riet dem Fußballer, sich noch am selben Tag in ein Krankenhaus zu begeben. Dort fertigte man eine Röntgenaufnahme in 2 Ebenen. Die Naht wurde als reizfrei bei intakter Motorik und Sensibilität beschrieben. Der behandelnde Arzt im Krankenhaus empfahl dem Kläger dringend eine antibiotische Therapie nebst einer Entfernung des Schleimbeutels (Bursektomie) unter Wiedereröffnung der vom Mannschaftsarzt vorgenommenen Wundnaht. Da der Kläger das weitere Vorgehen am darauffolgenden Tag mit dem Mannschaftsarzt besprechen wollte, musste sich der Klinikarzt bei seinen ärztlichen Bemühungen auf eine Tetanusprophylaxe beschränken. Am 01.11.2004 stellte sich der Kläger in der Praxis des Mannschaftsarztes vor. Dieser untersuchte den Kniebefund und veranlasste eine Kernspintomografie in einer radiologischen Praxis. Eine Ruptur der Kniebänder wurde dadurch ausgeschlossen. Anschließend wurde der Kläger wiederum vom Mannschaftsarzt in dessen Praxis an mehreren Tagen ambulant untersucht. Die erstmalige Befunderhebung durch einen weiteren Arzt am 10.11.2004 führte zu einer Wundrevision mit Sekundärnaht der Quadrizepssehne. An diesen Eingriff schloss sich ein von weiteren Operationen begleiteter stationärer Krankenhausaufenthalt bis zum 10.01.2005 an. Der weitere Verlauf war ebenfalls unbefriedigend, sodass der Kläger wegen des durch die Infektion verursachten dauerhaften Knieschadens seinem Beruf als Fußballspieler nicht mehr nachgehen kann.

Ursprünglich hatte der Kläger unter anderem neben dem Mannschaftsarzt auch noch das Krankenhaus sowie den im Krankenhaus behandelnden Klinikarzt wegen Fehlbehandlung auf materiellen und immateriellen Schadenersatz sowie auf Feststellung der Ersatzpflicht für entsprechende Zukunftsschäden in Anspruch genommen (Streitwert ca. 1450000 Euro).

Das Landgericht Trier hat die Klage des Fußballers abgewiesen. Mit der Berufung verfolgte der Kläger nur den Rechtsstreit gegen den Mannschaftsarzt sowie den Arzt, der die Wundrevision vorgenommen hatte, weiter. Das Oberlandesgericht Koblenz wies die Berufung des Fußballspielers einstimmig zurück (Beschluss OLG Koblenz vom 27.08.2012, Az.: 5 U 1510/11).

\section{Das erste Urteil: Kein Arztfehler}

Der Kläger hatte vorgetragen, die Erstversorgung in der Mannschaftskabine durch die zum Wundverschluss führende Naht der Verletzung sei grob fehlerhaft gewesen. Die gebotene sofortige Antibiose hätte der Erstbeklagte trotz eindeutiger Leitbefunde auch bei den ambulanten Nachuntersuchungen versäumt. Eine Bursektomie habe spätestens am 01.11.2004 erfolgen müssen. Wegen der am 10.11.2004 offen zutage liegenden Infektion im Kniegelenk sei die Naht der Quadrizepssehne zur Unzeit erfolgt.

Die beiden beklagten Ärzte erwiderten darauf, die Akutbehandlung sei unter dem bloßen Verdacht einer Bissverletzung erfolgt. Sofern der Biss einen Schleimbeutel

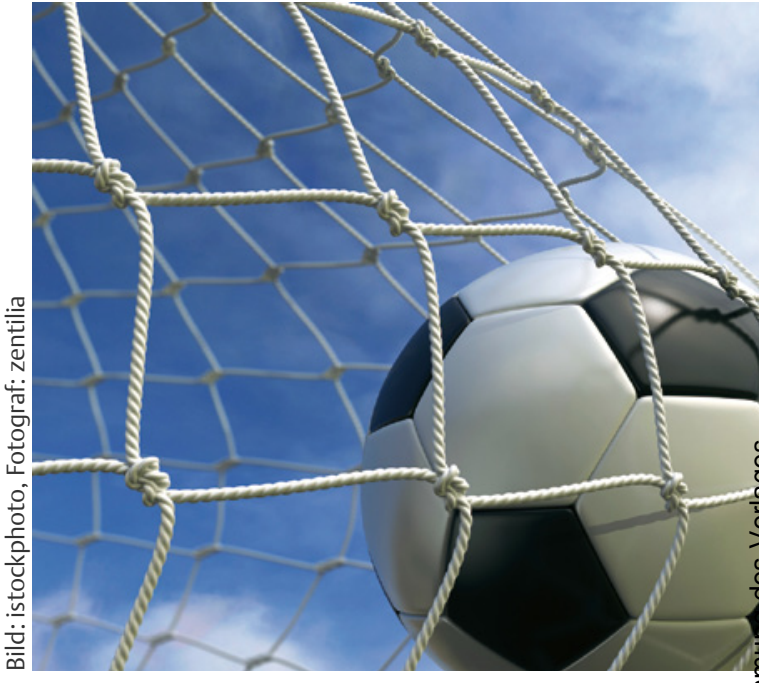

des Kniegelenks eröffnet habe, seien die schadensursächlichen Mundbakterien des Gegenspielers sofort dort eingedrungen. Die Primärnaht habe diesen Befund nicht richtunggebend verschlimmert. Im Übrigen habe der (frühere) beklagte Klinikarzt noch am selben Tag im Krankenhaus eindringlich darauf hingewiesen, dass wegen der hohen Infektionsgefahr derartiger Bissverletzungen eine sofortige Wundrevision mit Entfernung des Schleimbeutels und anschließender Antibiose erforderlich sei. Dies habe der Kläger abgelehnt, sodass er den weiteren Verlauf nunmehr nicht mit Erfolg den behandelnden Ärzten anlasten könne.

Das Landgericht hatte die Klage in erster Instanz mit der Begründung abgewiesen, die Frage, ob Bissverletzungen offen bleiben müssten oder vernäht werden dürften, sei umstritten. Kontrovers diskutiert werde auch, ob prophylaktisch Antibiotika verabreicht werden sollten. Angesichts dieses Meinungsstreits könne das Behandlungskonzept des Beklagten nicht als fehlerhaft angesehen werden. Die Untersuchung eines erstmals am 08.11.2004 genommenen Wundabstrichs sei nicht verspätet erfolgt, weil die Wunde bis dahin trocken gewesen sei. $\mathrm{Zu}$ Unrecht laste der Kläger den Beklagten an, eine Bursektomie versäumt zu haben. Die Annahme, dass eine Infektion ausbleibe, wenn man nicht chirurgisch interveniere, sei vertretbar gewesen. Im Übrigen habe der Erstbeklagte mit dem vom Kläger befolgten Rat, noch am selben Tag ein Krankenhaus aufzusuchen, das weitere Behandlungsgeschehen in die richtigen Bahnen gelenkt. Außerhalb eines Zeitfensters von 6 bis 12 Stunden nach einer derartigen Verletzung sei eine Bursekto- 
mie von derart zweifelhaftem Nutzen, dass der Erstbeklagte im November 2004 an seinem Konzept der konservativen Behandlung habe festhalten dürfen. Da der Sachverständige auch das weitere Vorgehen beider Beklagten als vertretbar angesehen habe, bestehe kein Ersatzanspruch des Klägers. Hiergegen wandte sich der Kläger mit der Berufung.

\section{Die Entscheidung des OLG: Grober Behandlungsfehler}

Welche Gefahr bei tierischen, erst recht jedoch bei menschlichen Bissverletzungen von einer Wundinfizierung durch Bakterien ausgeht, gehört nach Auffassung des Oberlandesgerichts (OLG) zum ärztlichen Standardwissen. Vor diesem Hintergrund hätte der erstbeklagte Mannschaftsarzt angesichts der Ungewissheit, wie tief die Verletzung reichte, bereits beim bloßen Verdacht einer Bissverletzung davon absehen müssen, die Wunde zu nähen. Im Nähen der Wunde sah das OLG, anders als das erstinstanzliche Gericht, einen groben Behandlungsfehler.

Soweit der Kläger behauptet, der Erstbeklagte habe seine therapeutische Aufklärungspflicht verletzt, weil er am 31.10.2004 versäumt habe, auf das Erfordernis einer Bursektomie und das dafür eröffnete schmale Zeitfenster hinzuweisen, folgt das OLG diesem Vortrag nicht.

Aufgrund ihrer oberflächlichen Lage unter der Haut sind die Schleimbeutel des Kniegelenks durch Infektionen über Verletzungen besonders gefährdet. Ob wegen einer derartigen Verletzung eine Bursektomie erforderlich ist, kann regelmäßig nur aufgrund einer umfassenden klinischen Untersuchung festgestellt und abschließend beurteilt werden. Für das Gericht stand daher außer Frage, dass Derartiges in der Mannschaftskabine eines Fußballstadions von einem Arzt nicht geleistet werden kann. Den Mannschaftsarzt traf daher nur die Pflicht, alsbald für eine ergänzende umfassende Befunderhebung zu sorgen. Derart ist er vorgegangen, indem er den Kläger in das Krankenhaus überwies.

\section{Aufklärung durch den Klinikarzt war ausreichend}

Dabei sei ohne Bedeutung, dass der Erstbeklagte bei der Überweisung weniger an eine Bursektomie als an das Erfordernis dachte, etwaige in der Wunde steckende abgebrochene Zähne bzw. Zahnreste des Gegenspielers des Klägers zu entfernen.
Die auf diese Weise angestoßene weitere Befundung führte nämlich zu einer richtigen Erkenntnis des früheren beklagten Klinikarztes, dass eine Bursektomie dringlich geboten war. Der Klinikarzt hatte nämlich bereits den Gegenspieler des Klägers behandelt, der sich in der Notfallaufnahme des Krankenhauses mit einem Kieferbruch eingefunden und dabei auch die durch den Aufprall gegen das Knie des Klägers ausgebrochenen Zähne präsentiert hatte. Vor diesem Hintergrund informierte der Klinikarzt den Kläger nach dessen Untersuchung darüber, dass „die Wunde so nicht bleiben könnte“, sie müsse dringend unter sterilen Bedingungen in einem Operationssaal geöffnet werden. Bei Schleimbeutelbeteiligung müsse eine Bursektomie vorgenommen werden. Die daran anschließende Mitteilung des Arztes, dass mit einem mehrwöchigen Ausfall zu rechnen sei, führte zu einer „immer ablehnenderen Haltung“ des Klägers. Selbst die Hinweise, dass das Infektionsrisiko „enorm hoch sei“ und welche weiteren ärztlichen Maßnahmen dringlich geboten seien, konnten den Kläger nicht zu einem Sinneswandel bewegen. Dadurch und durch die weiteren Bekundungen des Krankenhausarztes war nach Auffassung der Richter hinreichend belegt, dass dem Kläger all das an therapeutischer Aufklärung zuteil wurde, was vom Kläger in der Berufung bemängelt wurde.

\section{Fehler des Arztes wegen Behandlungsweigerung nicht mehr schadensursächlich}

Zwar waren die Richter der Auffassung, dass der grobe Behandlungsfehler des erstbehandelnden Mannschaftsarztes bei der Erstversorgung der Wunde (Vernähen) geeignet gewesen sei, eine Beweislastumkehr hinsichtlich des weiteren Kausalverlaufs herbeizuführen. Die Indizwirkung endete jedoch, als der Kläger sich wenig später nach der umfassenden Untersuchung, Befundung und Beratung durch den Krankenhausarzt entschloss, den sachgemäßen Behandlungsempfehlungen dieses Arztes trotz des akuten Handlungsbedarfs nicht zu folgen. Der Klinikarzt erkannte den Fehler des Erstbeklagten und teilte dem Kläger mit, dass es unerlässlich sei, die Naht sofort wieder zu öffnen, ein Debridement vorzunehmen und aufgrund der Abstrichuntersuchung eine gezielte Antibiose einzuleiten. Dass der Arzt damit bei dem Geschädigten auf taube Ohren stieß, war dem Um- stand geschuldet, dass der Kläger die mit einer sofortigen operativen Intervention bis hin zur Bursektomie verbundene mehrwöchige Berufsunfähigkeit und den daran anknüpfenden Entgang von Spielprämien scheute und stattdessen darauf vertraute, dass das vom Klinikarzt mit drastischen Worten geschilderte Infektionsrisiko sich nicht verwirklichen werde. Dadurch veränderte der umfassend über die Risiken informierte Kläger den weiteren Kausalverlauf richtunggebend, sodass der Fehler des Beklagten bei der Erstversorgung nicht mehr als schadensursächlich angesehen werden kann. In der Folge wurde die Berufung des Klägers zurückgewiesen.

Der Senat sah ein der Haftung der beiden beklagten Ärzte entgegenstehendes Eigenverschulden des Klägers darin, dass er sich am Abend des Unfalltages den sachgemäßen Therapieempfehlungen des zur Weiterbehandlung konsultierten Arztes verschloss. Die Nichtbefolgung der ärztlichen Ratschläge des Klinikarztes habe derartiges Gewicht, dass der Zurechnungszusammenhang zwischen der Pflichtwidrigkeit des Mannschaftsarztes und dem späteren Schaden nicht als erwiesen angesehen werden kann. Der hier zu entscheidende Sachverhalt wiese allerdings die Besonderheit auf, dass der Erstbeklagte durch seinen Fehler (Zunähen der Risswunde) einen Kausalverlauf in Gang gesetzt hatte, der alsbald, nämlich wenige Stunden später im Krankenhaus, in die medizinisch richtigen Bahnen gelenkt worden wäre, wenn der Kläger das dort vorgeschlagene weitere ärztliche Vorgehen nicht rundum abgelehnt hätte. Weil der Kläger selbst wie ein Zweitschädiger in den weiteren Geschehensablauf eingriff, indem er die sachgemäßen ärztlichen Ratschläge und Empfehlungen des Krankenhausarztes unbeachtet ließ, kann dem Erstschädiger ein Einstehenmüssen auch für diese Folgen billigerweise nicht zugemutet werden. Das Gericht war der Auffassung, dass diese rundum ablehnende Haltung des Klägers völlig ungewöhnlich und unsachgemäß war. Der Kläger habe selbst die alsbaldige Korrektur des Fehlers des Erstbeklagten abgelehnt und die damit verbundenen Risiken und damit auch die aus dem Fehler des Beklagten herrührende (weitere) Gefährdung „sehenden Auges“ in Kauf genommen. Der Kläger blendete nach Auffassung der Richter aus, dass die außerordentlich bedauerliche weitere Entwicklung nicht 
zwingend auf dem Fehler des Erstbeklagten (Vernähen der Risswunde) beruhen muss, sondern auch allein auf die Ausgangsverletzung zurückgehen kann, für deren schädliche Folgen der Erstbeklagte außerhalb seines Handlungsbeitrags nicht einzustehen hat. Der Fehler des Erstbeklagten ist nach dem Gesamtbild des vorliegenden Falles haftungsrechtlich im Bereich des Möglichen steckengeblieben. Ein grober Behandlungsfehler begründet ausnahmsweise keine Umkehr der Beweislast, wenn ein haftungsrechtlicher Zusammenhang zwischen dem Behandlungsfehler und dem eintretenden Schaden äußerst unwahrscheinlich ist. Dies könne auch dann der Fall sein, wenn der Geschädigte selbst in den weiteren Geschehensablauf dadurch eingreift, dass er die alsbaldige Fehlerkorrektur und die medizinisch gebotene Weiterbehandlung verweigert. Hierzu habe der Bundesgerichtshof angemerkt, dass eine Beweislastumkehr auch dann ausscheide, wenn der Patient durch sein Verhalten eine selbstständige Komponente für den Heilungserfolg vereitelt hat und dadurch in gleicher Weise wie der grobe Behandlungsfehler des Arztes dazu beigetragen hat, dass der Verlauf des Behandlungsgeschehens nicht mehr aufgeklärt werden kann. So habe es im vorliegenden Fall gelegen.

\section{Fazit}

Trotz vorliegenden groben Behandlungsfehlers wurden die verklagten Ärzte nicht verurteilt, da der Patient letztlich den Heilungserfolg selbst verhindert hat, indem er eindringlichen und sachgerechten ärztlichen Empfehlungen nicht gefolgt ist. Mitzunehmen aus diesem Fall ist sicherlich, dass eine gute Dokumentation (insbesondere auch über die Aufklärung, die in Haftungsprozessen immer als „Notanker" gerügt wird) hilfreich und gegebenenfalls prozessentscheidend sein kann. Eine solche ausführliche Dokumentation drängt sich insbesondere auf, wenn ersichtlich ist, dass der Patient dem ärztlichen Rat nicht folgen will.

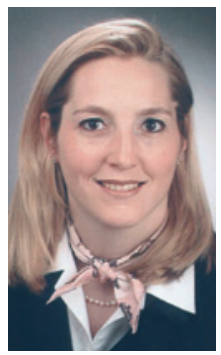

Korrespondenz

Dr. iur. Isabel Häser Rechtsanwältin

Ehlers, Ehlers und Partner Widenmayerstr. 29

80538 München 